Coexi st ence of transt hyretin- and $A \beta$ - type cer ebral anyl oi $d$ angi opat hy in a pat i ent with her edi tary transt hyret i n V3OM amyl oi dosi s

\begin{tabular}{|l|l|}
\hline 著者 & $\begin{array}{l}\text { Sakai Kenj i, Asakawa M wako, Takahashi } \\
\text { Ryoi chi, I shi da Chi ho, Nakamur a Ri t suko, } \\
\text { Hanaguchi Tsuyoshi, Ono Kenj i ro, I wasa Kazuo, } \\
\text { Yanada Nasahi to } \\
\text { 坂井 健二, 中村 律子, ?口 毅, 小野 賢二郎, 岩 } \\
\text { 佐 和夫, 山田 正仁 }\end{array}$ \\
\hline 著者別表示 & Jour nal of t he Neur ol ogi cal Sci ences \\
\hline $\begin{array}{l}\text { j our nal or } \\
\text { publ i cat i on t i t l e }\end{array}$ & 381 \\
\hline vol une & 144 146 \\
\hline page range & $2017-10-15$ \\
\hline year & ht t p: //doi . or g/10. 24517/00049681 \\
\hline URL & \multicolumn{1}{|c|}{ doi: 10.1016/.jns.2017.08.3240 }
\end{tabular}




\section{Coexistence of transthyretin- and A $\beta$-type cerebral amyloid angiopathy in a patient with hereditary transthyretin V30M amyloidosis}

Kenji Sakai, MD, $\mathrm{PhD}^{1}$, Miwako Asakawa, $\mathrm{MD}^{1}$, Ryoichi Takahashi, $\mathrm{MD}, \mathrm{PhD}^{1}$, Chiho Ishida, $\mathrm{MD}, \mathrm{PhD}^{2}$, Ritsuko Nakamura, $\mathrm{MD}, \mathrm{PhD}^{3}$, Tsuyoshi Hamaguchi, $\mathrm{MD}, \mathrm{PhD}^{1}$, Kenjiro Ono, $\mathrm{MD}, \mathrm{PhD}^{1,4}$, Kazuo Iwasa, $\mathrm{MD}, \mathrm{PhD}^{1}$, Masahito Yamada, $\mathrm{MD}, \mathrm{PhD}^{1}$

${ }^{1}$ Department of Neurology and Neurobiology of Aging, Kanazawa University Graduate School of Medical Sciences, Kanazawa, Japan

2 Department of Neurology, National Hospital Organization Iou Hospital, Kanazawa, Japan

${ }^{3}$ Department of Molecular and Cellular Pathology, Kanazawa University Graduate School of Medical Sciences, Kanazawa, Japan

${ }^{4}$ Department of Neurology, Showa University School of Medicine, Tokyo, Japan

Address correspondence to Dr. Kenji Sakai

Department of Neurology and Neurobiology of Aging, Kanazawa University Graduate School of Medical Science

13-1 Takara-machi, Kanazawa 920-8640, Japan

TEL: +81-76-265-2292

FAX: +81-76-234-4253

E-mail: ksakai@med.kanazawa-u.ac.jp

Keywords: transthyretin; amyloid $\beta$ protein; cerebral amyloid angiopathy; cross-seeding effect

Abbreviations: $\mathrm{A} \beta$, amyloid $\beta$ protein; apolipoprotein AI, apoAI; CAA, cerebral amyloid angiopathy; TTR, transthyretin 
Dear Editor,

Amyloidosis is characterized by deposition of specific amyloid proteins in the tissues.

Since different amyloid proteins have similar fibrillar structures, aggregation of distinct

amyloid proteins may exhibit cross-seeding effects on each other, resulting in fibril

formation of different amyloid proteins, as observed in experimental models and patients

[1-4]. Transthyretin (TTR) is a common cause of systemic amyloidosis affecting heart,

peripheral and autonomic nervous system, and eye. Moreover, cerebral amyloid

angiopathy (CAA) was demonstrated in some patients with systemic TTR amyloidosis

with mutations in the TTR gene [5]. CAA frequently occurs in the long-term clinical

course after liver transplantation [6]. Here, we describe an autopsy case of late-onset

systemic TTR amyloidosis with a V30M mutation in TTR showing severe CAA.

Interestingly, TTR as well as amyloid $\beta$ protein $(A \beta)$ were deposited in the cerebral

leptomeningeal and cortical blood vessel walls. Most of the TTR and A $\beta$ amyloid 
deposited independently on the vessel walls; however, a part of the vessel wall was occupied by a combination of TTR and A $\beta$. This case provides novel insights into the interaction of amyloidogenic proteins in humans.

An 84-year-old man with unremarkable family history developed lower and upper limb weakness, constipation, and anhydrosis at the age of 73. Dysesthesia of the extremities appeared later. His symptoms gradually deteriorated. At the age of 77 , he received a diagnosis of hereditary systemic TTR amyloidosis with TTR V30M mutation based on TTR-positive amyloid deposition in the sural nerve and genetic analysis. Oral administration of diflunisal was started [7]; however, his symptoms related to neuropathy and cardiac dysfunction deteriorated. Autonomic dysfunction gradually emerged.

Diflunisal was replaced with tafamidis at the age of 83. The patient presented disturbance of immediate memory followed by dementia with hallucination one year before the death. During the course of the disease, he did not show episodes of transient neurological 
symptoms due to central nervous system dysfunction (amyloid spells). He died of heart failure due to amyloidosis at the age of 84 . The total clinical course was 11 years.

Paraffin specimens of the tissues fixed with $10 \%$ buffered formalin were examined. Selected sections were immunostained using the ABC method with a Vectastain ABC kit (Vector, CA, USA). Double immunofluorescence was also performed (Fig. 1).

A general autopsy was performed 8.5 hours after the death. Systemic amyloid deposition was evident in the visceral organs. The brain and dura mater showed mild dilatation of the lateral ventricles and weighed 1,355 g before fixation. Macroscopic examinations revealed mild depigmentation in the substantia nigra and locus ceruleus. Microscopically, severe loss of myelinated fibers with TTR-positive amyloid deposition was demonstrated in the peripheral nerves. The dorsal root ganglia, sympathetic ganglia, skin, and pituitary also demonstrated considerable TTR-positive amyloid deposition (Fig. 
1a). In addition to TTR-positive amyloid deposition in the dura mater, leptomeninges,

subpial areas (Fig. 1b), and leptomeningeal blood vessels throughout the central nervous system, the cerebral cortical blood vessels showed CAA (Fig. 1c) comprising extensive TTR-positive amyloid deposition (Figs. 1b, d, g); however, no TTR-positive amyloid structures were observed in the brain and spinal cord parenchyma. Furthermore, widespread A $\beta$-positive senile plaques were observed (CERAD B; Braak stage B [8]; Thal phase 2). The cerebral cortical and leptomeningeal CAA (Fig. 1c) also consisted of A $\beta$ (Figs. 1e, h). Circumferential positivity for $A \beta$ was observed in some but not all leptomeningeal or cortical blood vessels in the cerebral cortices. A $\beta$ deposition in the vessels walls was more apparent in the occipital lobe. No A $\beta$-type CAA was observed in the cerebellum, brainstem, or spinal cord. Interestingly, most of the TTR and A $\beta$ deposited independently on the vessel walls, as analyzed using the double labeling method. A part of vessel wall was occupied by a combination of TTR and A $\beta$ (Figs. 1f, i). 
Regarding CAA-associated neuropathological findings, no obvious hemorrhages,

microinfarcts, or white matter damages were revealed. Although tau pathology was

confined to the medial temporal lobe (Braak \& Braak stage I; Braak AT8 stage II) [8],

Lewy body pathology was observed in the limbic systems and brainstem (Limbic stage)

[9].

In systemic TTR amyloidosis with specific mutations, mutant TTR proteins

produced by the choroid plexus may deposit in the leptomeningeal and cortical blood

vessel walls as in TTR-type CAA [5, 6]. Regardless of liver transplantation, central

nervous system clinical symptoms frequently occurred in patients with systemic TTR

amyloidosis with a V30M mutation in the TTR gene [6]. Interestingly, coexistence of

TTR- and A $\beta$-type CAA in a single case, as found in this patient, has never been reported so far. Since our patient demonstrated manifestations regarding TTR amyloidosis at the age of 73, this case also developed A $\beta$-type CAA, which is commonly observed in elder 
people. A $\beta$-type CAA has been considered to be consequence of a failure of intramural periarterial drainage owing to modification of the cerebrovascular basement membrane [10]. Although TTR deposition in the vessel walls could damage this perivascular drainage pathway, it is unlikely that widespread occurrence of vascular TTR deposition exerted an influence on development of $A \beta$-type CAA in this case due to the fact that distribution of A $\beta$-type CAA was an ordinary manner, such as a predominance in the occipital lobe.

Regarding cause of dementia in our patient observed one year before the death, Lewy body pathology could make a profound impact on the dementia the result of less severity of the tau pathology [9]. Meanwhile, moderate-to-very severe A $\beta$-type neocortical CAA is associated with decreased perceptual speed after adjusting for comorbid parenchymal Alzheimer’s disease pathology and Lewy body pathology [11]. Moreover, several specific TTR gene mutations present central nervous system 
manifestations including dementia [12]. Although assessments of the cognitive

dysfunction in our patient were inadequate, severe CAA observed in this case, which is comprised of TTR- and A $\beta$-type, might be related to development of dementia.

Several in vivo [1, 3, 4] and in vitro [2] evidence suggest cross-seeding effects of amyloidogenic proteins. Amylin and A $\beta$ show cross-seeding effects and may deposit as a combination of amyloid in the human brain parenchyma and blood vessels [1, 3]. Apolipoprotein AI (ApoAI) and TTR co-localized in amyloid deposits of the biopsied tissues in a patient with apoAI amyloidosis with Leu78His mutation [4]. Moreover, hybrid amyloid fibril comprising $\mathrm{A} \beta$ and amylin has been previously generated in vitro [2]. Our findings, however, that most of the TTR and A $\beta$ in the vessel walls deposited independently (Figs. 1f, i) indicate that cross-seeding of TTR and A $\beta$ is less likely in humans. TTR, a protein in the cerebrospinal fluid, was reported to bind to A $\beta$ and inhibit A $\beta$ aggregation in vitro [13]. An in vivo experiment using human kidney also revealed 
that TTR plays an important role in the sequestration of $A \beta$ to prevent $A \beta$ aggregation and amyloid formation in the kidney [14]. On the other hand, a study using Alzheimer's disease mice model demonstrated that TTR is associated with increase of A $\beta$ deposition on the vessel walls [15]. Due to the fact that experimental and in vivo human evidence of association between TTR and A $\beta$ is inconsistent, further studies are essential to clarify interaction between TTR and A $\beta$ in the human brain.

In conclusion, we describe an autopsy case of late-onset systemic TTR amyloidosis with a V30M mutation in TTR showing coexistence of TTR- and A $\beta$-type CAA in the central nervous system. Independent deposition of the TTR and A $\beta$ amyloid on the vessel walls provides novel insights into the interaction of amyloidogenic proteins, including TTR and A $\beta$, in humans. This case raises the need for specific amyloid tracers for in vivo diagnosis. 


\section{Acknowledgments}

The authors thank Professor Akishi Ooi (Department of Molecular and Cellular

Pathology, Kanazawa University Graduate School of Medical Sciences, Japan) for

comments on the pathological diagnosis and also Yumiko Kakuda, Ritsuko Goto, Miyuki

Honda, and Natsu Yamada for technical assistance.

\section{Conflict of interest}

There is no conflict of interest related to the manuscript.

\section{Funding}

This research did not receive any specific grant from funding agencies in the public,

commercial, or not-for-profit sectors. 


\section{References}

1. Jackson K, Barusibe GA, Diaz E, Jin L, DeCarli C, Despa F. Amylin deposition in the brain: a second amyloid in Alzheimer disease. Ann Neurol 2013; 74: 517-526

2. Hu R, Zhang M, Chen H, Jiang B, Zheng J. Cross-seeding interaction between $\beta$-amyloid and human islet amyloid polypeptide. ACS Chem Neurosci 2015; 6: 17591768

3. Oskarsson ME, Paulsson JF, Schultz SW, Ingelsson M, Westmark P, Westmark GT. In vivo seeding and cross-seeding of localized amyloidosis: a molecular link between type 2 diabetes and Alzheimer disease. Am J Pathol 2015; 185: 834-846

4. de Sousa MM, Vital C, Ostler D, Fernandes R, Pouget-Abadie J, Carles D, Saraiva MJ. Apolipoprotein AI and transthyretin as components of amyloid fibrils in a kindred with apoAI Leu178His amyloidosis. Am J Pathol 2000; 156: 1911-1917

5. Ushiyama M, Ikeda S, Yanagisawa N. Transthyretin-type cerebral amyloid angiopathy in type I familial amyloid polyneuropathy. Acta Neuropathol 1991; 81: $524-528$

6. Maia LF, Magalhães R, Freitas J, Taipa R, Pires MM, Osório H, Dias D, Pessegueiro 
H, Correia M, Coelho T. CNS involvement in V30M transthyretin amyloidosis:

clinical, neuropathological and biochemical findings. J Neurol Neurosurg Psychiatry 2015; 86: 159-167

7. Takahashi R, Ono K, Shibata S, Nakamura K, Komatsu J, Ikeda Y, Ikeda T, Samuraki M, Sakai K, Iwasa K, Kayano D, Yamada M. Efficacy of diflunisal on autonomic dysfunction of late-onset familial amyloid polyneuropathy (TTR Val30Met) in a Japanese endemic area. J Neurol Sci 2014; 345: 231-235

8. Braak H, Braak E. Neuropathological staging of Alzheimer-related changes. Acta Neuropathol 1991;82:239-259

9. McKeith IG, Dickson DW, Lowe J, et al. Diagnosis and management of dementia with Lewy bodies: third report of the DLB consortium. Neurology 2005;65:18631872

10. Carare RO, Hawkes CA, Jeffrey M, Kalaria RN, Weller RO. Cerebral amyloid angiopathy, prion angiopathy, CADASIL and the spectrum of protein elimination failure angiopathies (PEFA) in neurodegenerative disease with a focus on therapy. Neuropathol Appl Neurobiol 2013;39:593-611 
11. Arvanitakis Z, Leurgans SE, Wang Z, Wilson RS, Bennett DA, Schneider JA.

Cerebral amyloid angiopathy pathology and cognitive domains in older persons. Ann

Neurol 2011; 69: 320-327

12. Nakamura M, Yamashita T, Ueda M, Obayashi K, Sato T, Ikeda T, Washimi Y, Hirai

T, Kuwahara Y, Yamamoto MT, Uchino M, Ando Y. Neuroradiologic and

clinicopathologic features of oculoleptomeningeal type amyloidosis. Neurology

2005; 65: 1051-1056

13. Liu L, Murphy RM. Kinetics of inhibition of $\beta$-amyloid aggregation by transthyretin.

Biochemistry 2006; 45: 15702-15709

14. Tsuzuki K, Fukatsu R, Yamaguchi H, Tateno M, Imai K, Fujii N, Yamauchi T.

Transthyretin binds amyloid $\beta$ peptides, $A \beta 1-42$ and $A \beta 1-40$ to form complex in the autopsied human kidney - possible role of transthyretin for $\mathrm{A} \beta$ sequestration.

Neurosci Lett 2000; 281: 171-174

15. Wati H, Kawarabayashi T, Matsubara E, Kasai A, Hirasawa T, Kubota T, Harigaya Y, Shoji M, Maeda S. Transthyretin accelerates vascular A $\beta$ deposition in a mouse model of Alzheimer's disease. Brain Pathol 2009; 19: 48-57 


\section{Figure legend}

Figure 1 Postmortem neuropathological findings.

(a) The dorsal root ganglion shows widespread Congo red-positive amyloid deposition.

(b) Amyloid deposits immunolabeled for transthyretin (TTR) are observed around the lumbar spinal cord and leptomeningeal blood vessels.

(c) Congo red-positive amyloid deposition (cerebral amyloid angiopathy, CAA) is observed in the leptomeningeal and cerebral cortical blood vessels.

(d-f) Serial sections demonstrate that CAA comprises both TTR (d) and amyloid $\beta$ protein (A $\beta$ ) (e) deposition. Most of the TTR and A $\beta$ deposits are separately distributed in the vessel walls (f).

(g-i) In another leptomeningeal blood vessel, a part of the vessel wall is occupied by combination of TTR and A $\beta$ amyloid (arrow).

The primary antibodies used are as follows: $\mathrm{A} \beta$ (4G8; mouse monoclonal; Covance; 1:5000) and prealbumin (TTR) (rabbit monoclonal; Abcam; 1:100). Double immunofluorescence is performed using primary antibodies to $A \beta$ (4G8; 1:500), prealbumin (1:50), and secondary antibodies conjugated with Alexa 488 (Invitrogen, 1:400, green) or Alexa 568 (1:400, red) fluorochromes, respectively. 
Congo red (a, c); immunostaining for TTR (b, d, g), and A $\beta$ (e, h). Merged images of TTR and A $\beta$ (f, i). Scale Bars = $200 \mu \mathrm{m}$ (a); 1 mm (b); $100 \mu \mathrm{m}$ (c-i). 


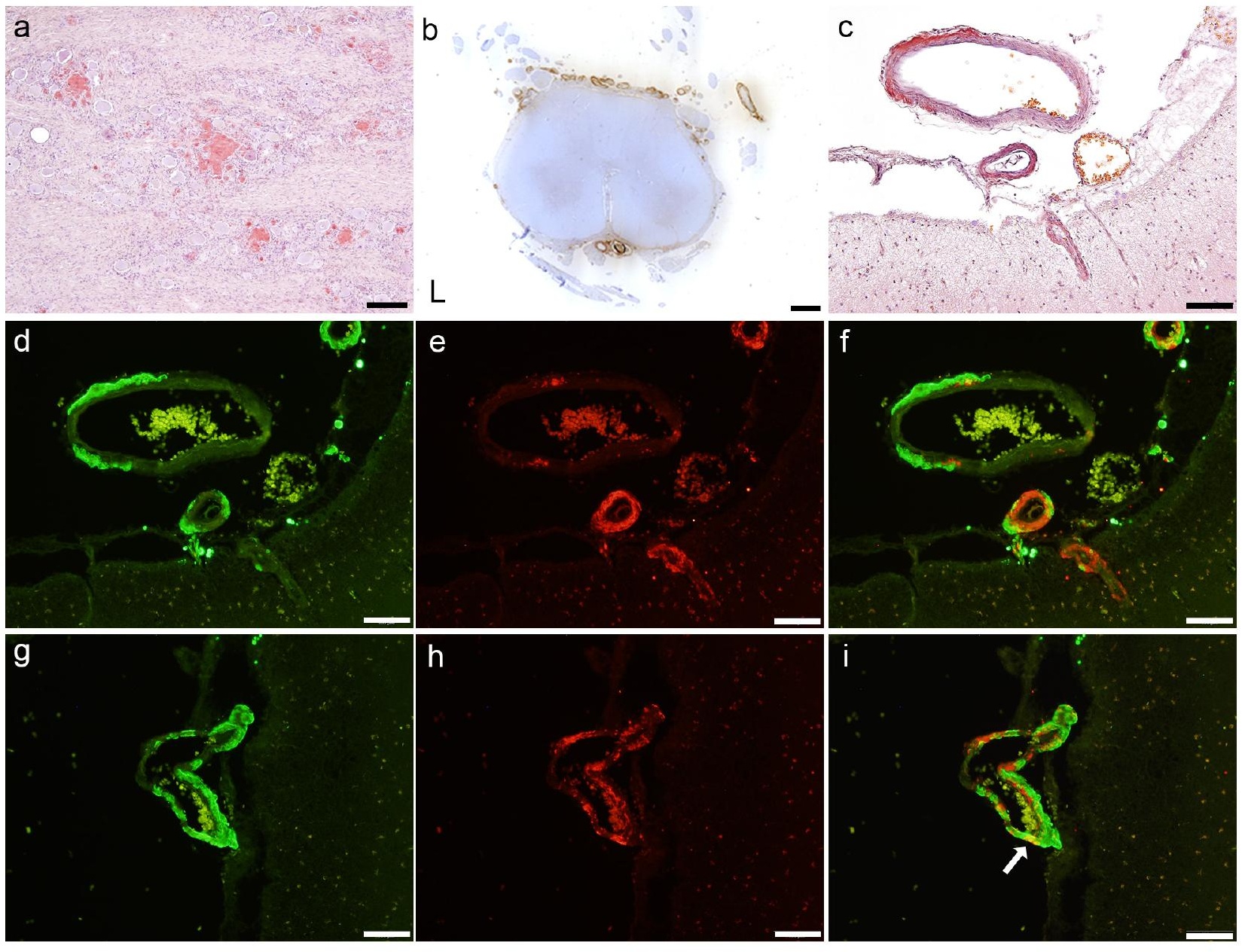

